\title{
Magnetic response of non-interacting and interacting electrons in a Möbius strip
}

\author{
Madhumita Saha ${ }^{1}$ and Santanu K. Maiti, ${ }^{1, *}$ \\ ${ }^{1}$ Physics and Applied Mathematics Unit, Indian Statistical Institute, \\ 203 Barrackpore Trunk Road, Kolkata-700 108, India
}

\begin{abstract}
We investigate characteristic features of both non-interacting and interacting electrons in a Möbius strip, the simplest possible one-sided topological system, in presence of an Aharonov-Bohm flux $\phi$. Using Hartree-Fock mean field theory we determine energy eigenvalues for the interacting model, while for the non-interacting system an analytical prescription is given. The interplay between longitudinal and vertical motions of electrons along with on-site Hubbard interaction yield several anomalous features of persistent current associated with energy-flux characteristics. The variation of current with system size and its temperature dependences are also critically examined. Current is highly sensitive to both these two factors, and we find that for a particular system size it decreases exponentially with temperature. Our analysis can be helpful in investigating electronic transport through any non-trivial topological material.

PACS numbers: 73.23.Ra, 71.27.+a, 73.23.-b
\end{abstract}

\section{INTRODUCTION}

The physics of topologically non-trivial materials can offer a new route to design conventional electronic devices. $\mathrm{NbSe}_{3}$ Möbius strip is one such possible geometry that was developed experimentally by Tanda et al $\stackrel{\underline{1}}{\text { in }}$ 2002 considering niobium and selenium compound. It is a one-sided topological system, unlike a regular cylinder, which is built by twisting a two-leg ladder and connecting its two ends. Several spectacular features are

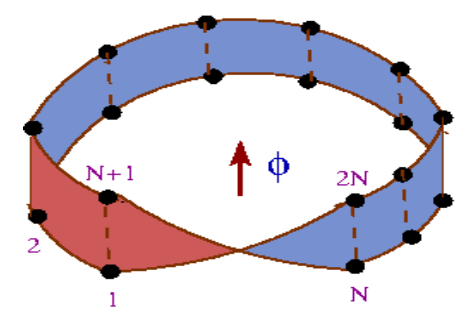

FIG. 1: (Color online). One-fold twisted Möbius strip threaded by an AB flux $\phi$ where the filled black circles correspond to the lattice sites.

exhibited by a twisted Möbius geometry. One simplest and realistic example of such system can be the Möbius graphene strip. In 2009 Guo et al $\stackrel{2}{2}$ have shown that a Möbius graphene strip with a zigzag edge behaves as a topological insulator with a gapped bulk and a robust metallic surface. Due to the significant potential applications, topological insulating materials have been under great focus both theoretically and experimentally, and the Möbius graphene strip is the suitable candidate for it. After successful fabrication of $\mathrm{NbSe}_{3}$ inorganic conductor much attention has been given to explore electronic properties of different topological shape conductors ${ }^{3-\underline{7}}$, expecting their strange contributions in designing nanoelectronic devices. For a purposeful design, a clear understanding of electronic behavior is highly important, and for isolated conductors having single or multiple loops it can suitably be done by analyzing magnetic response in presence of Aharonov-Bohm (AB) flux $\phi$. Due to flux $\phi$, a Berry phase is introduced in moving electrons which breaks time reversal symmetry and results a nonvanishing charge current. This is the so-called persistent current, an obvious demonstration of $\mathrm{AB}$ effect, and was first proposed by Büttiker et al 을 during early 80's. Following this pioneering work, substantial theoretical and experimental works ${ }^{9}-27$ have been done along this line to understand different aspects of persistent current and other related issues in isolated conducting loops. In particular, the physics of quantum rings has always been the subject of intense research due to its potential applications in designing electronic, spintronic, optoelectronic and information processing devices. The innovative recent advances in experimental and theoretical physics of quantum rings are available in Ref 28 .

A similar kind of non-decaying circular current is also obtained in other context ${ }^{29}-32$ where a ring-shaped conductor is connected with source and drain electrodes. Exploiting the effect of quantum interference among electronic waves passing through different branches of a conducting junction (viz, source-conductor-drain junction) one can establish a net current, in presence of a finite bias, which exhibits several interesting results. These features are not discussed here as they are beyond the scope of our present work, and hopefully we will reproduce them in our forthcoming work.

Now, the works involving flux-driven persistent current in isolated systems are mostly confined to simple loop geometries like single-channel rings, multi-channel cylinders, graphene rings, nanotubes, array of rings to name a few ${ }^{-2}-20$. Whereas, very few works are available where twisted geometries have been taken into account. For instance, in 2003 Cohen et al $\underline{\underline{33}}$ have studied the behavior of persistent current for a non-interacting Möbius strip and latter in 2009 considering spinless interacting electrons Mori and Ota ${ }^{34}$ have investigated electronic behavior in this particular geometry. In 2010 Lassen et $a l \stackrel{35}{n}$ have investigated finite-thickness effects consider- 
ing different sized Möbius structures in presence of hydrostatic strain and explored several significant results. Latter in $2012 \mathrm{Li}$ and Ram-Mohan have done ${ }^{36}$ a detailed study on a Möbius ring and revealed significant new ideas. In the same year, Fomin et al. have shown the delocalization-to-localization ${ }^{37}$ transition taking an inhomogeneous Möbius ring which certainly highlights a great challenge in the current era of nanofabrication. Though the studies involving electronic properties in different topological conductors have generated a wealth of literature knowledge, to the best of our knowledge, no one has reported the phenomenon of persistent current in presence of on-site Coulomb interaction which always gives non-trivial features and certainly it demands further study.

In the present work we essentially focus on magnetic response of interacting electrons in a Möbius strip where the interaction parameter is treated within a HartreeFock (HF) mean field (MF) level ${ }^{38-42}$. The interplay between longitudinal and vertical motions of electrons along with on-site Hubbard repulsion exhibits several anomalous features those are interesting and important too. Restricting electron motion along the vertical direction conventional $\phi_{0}(=c h / e$, the elementary flux-quantum $)$ periodicity of current can be changed to $\phi_{0} / 2$, which was reported in the literature ${ }^{33}$ considering non-interacting Möbius geometry. Apart from this, $\phi_{0} / 2$ periodic current can also be noticed depending on system size and filling factor even when the vertical motion is allowed. In addition we find that current is highly sensitive to system size and temperature. Both these effects are analyzed in detail. Though our main intention of the present work is to study magnetic response of interacting Möbius strip, for the sake of completeness here we also discuss characteristic properties of non-interacting electrons for which energy eigenvalues and persistent currents are evaluated analytically. In absence of vertical hopping we can find closed analytical form of net current for any arbitrary filling. Quite interestingly we see that for all odd number of electrons current gets a single expression, and similarly, for even number of electrons it gets another form.

Our work is organized as follows. In Sec. II we present the model and the method for theoretical calculations. The results are presented in Sec. III, and at the end we conclude in Sec. IV.

\section{MODEL AND THEORETICAL FORMULATION}

Figure 1 displays a $2 N$-site Möbius strip which is formed by twisting a two-leg ladder, where each leg contains $N$ atomic sites, and connecting its two ends. A magnetic flux $\phi$, measured in unit of $\phi_{0}(=c h / e)$ is allowed to pass through the centre of the strip such that electrons move in a field-free region. To describe this model we use tight-binding framework and in presence of on-site Hubbard interaction it reads as,

$$
\begin{aligned}
\mathbf{H}_{M} & =\sum_{\substack{j=1 \\
\sigma=\uparrow, \downarrow}}^{2 N} \epsilon_{j, \sigma} c_{j, \sigma}^{\dagger} c_{j, \sigma}+t \sum_{\substack{j=1 \\
\sigma=\uparrow, \downarrow}}^{2 N}\left[e^{i \theta} c_{j, \sigma}^{\dagger} c_{j+1, \sigma}+\text { h.c. }\right] \\
& +t_{\perp} \sum_{\substack{j=1 \\
\sigma=\uparrow, \downarrow}}^{2 N} c_{j, \sigma}^{\dagger} c_{j+N, \sigma}+U \sum_{j=1}^{2 N} c_{j, \uparrow}^{\dagger} c_{j, \uparrow} c_{j, \downarrow}^{\dagger} c_{j, \downarrow}
\end{aligned}
$$

where the meanings of different symbols are explained as follows. $\epsilon_{j, \sigma}$ is the on-site energy of an electron at $j$ th site with spin $\sigma(\uparrow, \downarrow)$ and $c_{j, \sigma}^{\dagger}\left(c_{j, \sigma}\right)$ represents the creation (annihilation) operator. $t$ represents the nearestneighbor hopping integral for the longitudinal motion of electrons, while it is $t_{\perp}$ for the vertical motion. $\theta(=$ $\left.2 \pi \phi / N \phi_{0}\right)$ is the phase factor due to $\mathrm{AB}$ flux $\phi$ and $U$ gives the on-site Hubbard interaction strength. Here we impose the boundary condition $j+2 N=j$.

For $U=0$ the system becomes a non-interacting one, and under this situation all the features can be analyzed quite easily. Whereas for the interacting case (viz, $U \neq$ $0)$ it is very hard to find energy eigenvalues directly by diagonalizing the full many-body Hamiltonian (Eq. 1), in particular for large $N$ and higher number of up and down spin electrons ${ }^{14.41}$. Therefore, to find the energy eigenvalues in the present article we use Hartree-Fock mean field approximation which essentially decouples the many-body Hamiltonian into two non-interacting ones associated with up and down spin electrons $\underline{40} \underline{-42}$. The effective MF Hamiltonian gets the form:

$$
\mathbf{H}_{M}^{M F}=\mathbf{H}_{M, \uparrow}+\mathbf{H}_{M, \downarrow}-U \sum_{j=1}^{2 N}\left\langle n_{j, \uparrow}\right\rangle\left\langle n_{j, \downarrow}\right\rangle
$$

where $\left\langle n_{j, \sigma}\right\rangle=\left\langle c_{j, \sigma}^{\dagger} c_{j, \sigma}\right\rangle$. The non-interacting Hamiltonians $\left(\mathbf{H}_{M, \uparrow}\right.$ and $\left.\mathbf{H}_{M, \downarrow}\right)$ are parameterized with effective site energies, while the other parameters $\left(t\right.$ and $\left.t_{\perp}\right)$ associated with electron hopping remain unchanged. The effective on-site energies are $\epsilon_{j, \uparrow}+U\left\langle n_{j, \downarrow}\right\rangle$ and $\epsilon_{j, \downarrow}+U\left\langle n_{j, \uparrow}\right\rangle$, respectively, for up and down spin electrons.

From these decoupled non-interacting Hamiltonians we can easily determine energy eigenvalues and evaluate net energy of the system at absolute zero temperature $(T=$ $0 \mathrm{~K}$ ) from the relation

$$
E_{0}(\phi)=\sum_{i=1}^{N_{\uparrow}} E_{M, \uparrow}^{i}+\sum_{i=1}^{N_{\downarrow}} E_{M, \downarrow}^{i}-U \sum_{i=1}^{2 N}\left\langle n_{i, \uparrow}\right\rangle\left\langle n_{i, \downarrow}\right\rangle
$$

where $E_{M, \uparrow}^{i}$ 's and $E_{M, \downarrow}^{i}$ 's are the energy eigenvalues of the non-interacting Hamiltonians. $N_{\uparrow}$ and $N_{\downarrow}$ correspond to the number of up and down spin electrons, respectively, which fix the total number of electrons in the system $N_{e}=N_{\uparrow}+N_{\downarrow}$. For finite temperature, this relation (Eq. 3) gets modified where the contributions from all energy levels are taken into account with proper weight factor governed by the Fermi-Dirac distribution function. 
In this case we have to specify chemical potential $\mu$, instead of $N_{e}$.

Once $E_{0}(\phi)$ is determined, the persistent current is obtained from the expression $9,11,12$

$$
I(\phi)=-c \frac{\partial E_{0}(\phi)}{\partial \phi} .
$$

Thus taking the first order derivative of ground state energy with respect to flux $\phi$ persistent current is determined, and it is the general expression ${ }^{9}$ for evaluating persistent current in a system whether it is characterized by fixed number of electrons $N_{e}$ or constant chemical potential $\mu$. At absolute zero temperature, $E_{0}(\phi)$ is determined by taking the sum of lowest $N_{\uparrow}$ and $N_{\downarrow}$ energy eigenvalues associated with total number of electrons $N_{e}$ ( $\left.=N_{\uparrow}+N_{\downarrow}\right)$ or chemical potential $\mu$ for each value of $\phi$, as other energy levels are not occupied by electrons. While, for the case of non-zero temperature, finite occupation probabilities are obtained for all energy levels (they are different depending on the energy eigenvalues). Here we characterize the system by constant $\mu$ (for a specific $\mu, N_{\uparrow}$ and $N_{\downarrow}$ are determined self-consistently), instead of $N_{e}$, and calculate the occupation probabilities of all the energy levels having energies $E_{M, \uparrow}^{i}$ and $E_{M, \downarrow}^{i}$. Then multiplying the occupation probability and associated energy eigenvalue of each level and taking the sum of this product over all energy levels we calculate $E_{0}(\phi)$.

\section{RESULTS AND DISCUSSION}

Below we present our results which include characteristic features of non-interacting and interacting electrons in a Möbius strip. Throughout the analysis we measure energy parameters in unit of electron-volt $(\mathrm{eV})$ and calculate current in unit of et/h, where $e$ and $h$ are the fundamental constants. We set $c=1$.

\section{A. Zero temperature limit}

Let us begin with non-interacting Möbius strip setting its temperature to zero. For non-interacting spinless case, the TB Hamiltonian reads as,

$$
\begin{aligned}
\mathbf{H}_{M}= & \sum_{j=1}^{2 N} \epsilon_{j} c_{j}^{\dagger} c_{j}+t \sum_{j=1}^{2 N}\left[e^{i \theta} c_{j}^{\dagger} c_{j+1}+h . c .\right] \\
& +t_{\perp} \sum_{j}^{2 N} c_{j}^{\dagger} c_{j+N}
\end{aligned}
$$

where different terms carry identical meanings as discussed above. For a perfect Möbius strip $\epsilon_{j}$ 's are identical and we can set them to zero, for simplification. Under this situation the energy eigenvalues are obtained from the relation:

$$
E_{n}=2 t \cos \left[\frac{\pi}{N}\left(n+\frac{2 \phi}{\phi_{0}}\right)\right]+t_{\perp} \cos [n \pi]
$$

where $n$ is restricted within the range $-N \leq n<N$. From this relation we can calculate the current carried by $n$th eigenstate as

$$
I_{n}=-\frac{\partial E_{n}(\phi)}{\partial \phi}=\frac{4 \pi e t}{N h} \sin \left[\frac{\pi}{N}\left(n+\frac{2 \phi}{\phi_{0}}\right)\right]
$$

and thus for $N_{e}$ electron system net current becomes $I(\phi)=\sum_{n=1}^{N_{e}} I_{n}(\phi)$.

When $t_{\perp}=0$, the net current gets the form:

$$
\begin{array}{rr}
I(\phi)=-2 I_{0} \frac{\sin \left[\frac{\pi}{2 N}\left(\frac{4 \phi}{\phi_{0}}\right)\right]}{\sin \left[\frac{\pi}{2 N}\right]} ; \quad-0.25 \leq \frac{\phi}{\phi_{0}}<0.25 \\
\text { for odd } N_{e} \\
=-2 I_{0} \frac{\sin \left[\frac{\pi}{2 N}\left(\frac{4 \phi}{\phi_{0}}-1\right)\right]}{\sin \left[\frac{\pi}{2 N}\right]} ; \quad 0.0 \leq \frac{\phi}{\phi_{0}}<0.5 \\
\text { for even } N_{e}
\end{array}
$$

where $I_{0}=\frac{e v_{f}}{L}, v_{f}$ being the Fermi velocity determined at $k=k_{f}$ (Fermi wave vector) and $L=2 N a$ ( $a$ is the lattice spacing). For non-zero $t_{\perp}$ we cannot find any such closed form of current for a wide flux window, like Eq. 8 . for arbitrary electron filling. It is apparent from Eq. 6 that the term involving $t_{\perp}$ does not contain any flux dependent term, so that its contribution on persistent current should be lifted after differentiating the energy with respect to flux (Eq. 7), and thus, one can also expect the closed analytical form of current like Eq. 8 for the situation when $t_{\perp}$ is finite. But this is not true, as the closed analytical form of current is obtained only when the contributing energy levels (indexed by $n$ ) appear sequentially i.e., $n=0$ for $N_{e}=1 ; n=0,-1,1$ for $N_{e}=3$, and similarly for even $N_{e}$. The appearance of contributing energy levels within the above mentioned flux range for odd and even $N_{e}$ can be easily understood from the energy-flux spectrum given below (see Fig. 2). This sequence of $n$ is not followed when $t_{\perp}$ is finite, as it changes the pattern of energy-flux levels (see Fig. 4) and that is reason behind the consideration of $t_{\perp}=0$ to get closed analytical form of persistent current given in Eq. 8 .

Based on the above analytical expressions (Eqs. 6, 8] we can easily characterize energy levels and current-flux spectra. In Fig. 2 the full energy spectrum is shown for a 10 -site Möbius strip considering $t=-1$ and $t_{\perp}=0$. Multiple crossings among different energy levels are obtained, yielding degeneracies, at different values of flux like $\phi=0, \pm m \phi_{0} / 4, \pm m \phi_{0} / 2$ and $\pm m \phi_{0}$, where $m$ is an integer. All these energy levels exhibit $\phi_{0} / 2$ fluxquantum periodicity, unlike conventional $\phi_{0}$ periodicity obtained in a regular cylinder. The reason is that for $t_{\perp}=0$ an electron which moves along the strip encloses a flux $2 \phi_{0}$, instead of $\phi_{0}$, when it comes back to its initial position as it encircles the loop twice. This behavior gets reflected in current-flux characteristics. To illustrate it in Fig. 3 we present the variation of persistent current in a 
200-site Möbius strip with $t=-1$ and $t_{\perp}=0$, considering odd and even number of electrons. Current exhibits saw-tooth like variation where sharp transitions at different $\mathrm{AB}$ fluxes are associated with the crossing of energy levels. A clear signature of $\phi_{0} / 2$ periodicity is observed from these $I-\phi$ spectra (Fig. 33).

The energy spectrum gets significantly modified with the inclusion of $t_{\perp}$. It is shown in Fig. 4 where we plot distinct energy levels for a 10-site Möbius strip considering

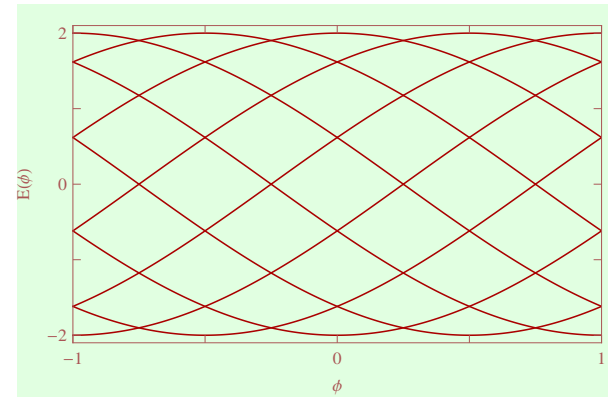

FIG. 2: (Color online). Energy-flux characteristics of a 10site non-interacting $(U=0)$ Möbius strip with $t=-1$ and $t_{\perp}=0$.
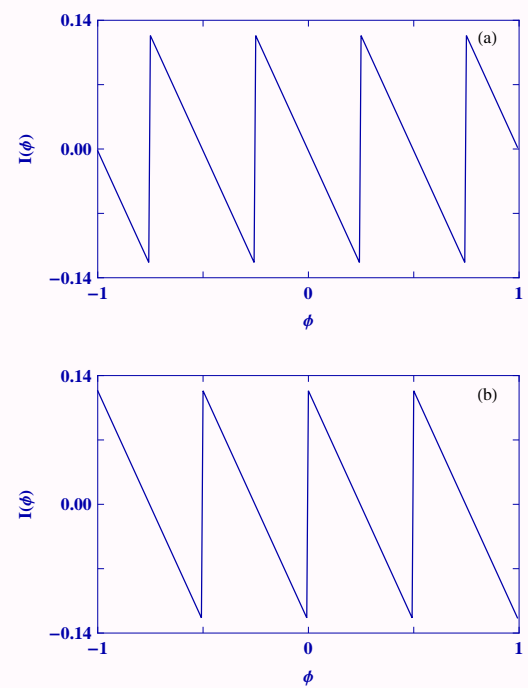

FIG. 3: (Color online). Persistent current as a function of flux $\phi$ in a 200-site Möbius strip, where (a) and (b) correspond to $N_{e}=95$ and 96 , respectively. The other physical parameters are: $U=0, t=-1$ and $t_{\perp}=0$.

$t=-1$ and $t_{\perp}=-0.8$. An overlap region appears across the energy band centre, unlike a regular single-channel ring, which is responsible in producing anomalous kinklike structure in persistent current provided the Fermi energy lies within this energy zone. The appearance of this overlap region can be explained from the energy expression given in Eq.6. Depending on the value of energy level index $n$, Eq. 6] gets splitted into two relations as

$$
E_{n}^{1}(\phi)=-t_{\perp}+2 t \cos \left[\frac{\pi}{N}\left(n+\frac{2 \phi}{\phi_{0}}\right)\right]
$$

and

$$
E_{n}^{2}(\phi)=t_{\perp}+2 t \cos \left[\frac{\pi}{N}\left(n+\frac{2 \phi}{\phi_{0}}\right)\right]
$$

These two expressions produce two energy sub-bands and their overlap is essentially controlled by $t_{\perp}$. For finite strength of $t_{\perp}$, electron does not acquire $2 \phi_{0}$ flux to reach to its initial starting point, rather it encloses $\phi_{0}$ flux, which results energy levels $\phi_{0}$ periodic (see Fig. 41).

This features enables us to characterize current-flux spectra given in Fig. 5 where we present the variation of current as a function of flux $\phi$ for a 200-site Möbius strip considering $t=-1$ and $t_{\perp}=-0.8$, where (a) and (b) correspond to $N_{e}=95$ and 96 , respectively. For odd $N_{e}$, a kink-like structure is observed across $\phi= \pm 0.5$, while

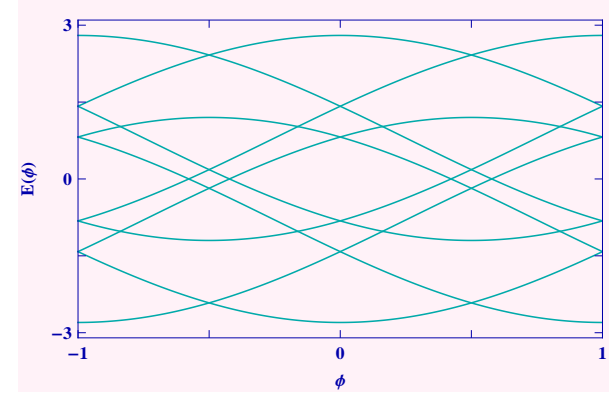

FIG. 4: (Color online). Energy spectrum for a 10-site noninteracting Möbius strip with $t=-1$ and $t_{\perp}=-0.8$.

for even $N_{e}$ it is not separable from the other parts due to increased kink height. In both these two fillings current exhibits conventional $\phi_{0}$ periodicity, following $E$ - $\phi$ curves (Fig. 4).

Though $\phi_{0}$ periodic current is naturally expected for finite value of $t_{\perp}$, but under a certain condition current yields half-flux quantum $\left(\phi_{0} / 2\right)$ periodicity. It is the halffilled band case with even $N$. This is exactly what we present in Fig. 6] where current is computed for a 200site (i.e., $N=100$ ) Möbius strip in the half-filled limit. Here it is important to note that for a regular cylinder (untwisted geometry), $\phi_{0} / 2$ periodicity is also observed at half-filling but $N$ should be $o d d$.

The results analyzed so far are worked out for noninteracting Möbius strips, and now we focus our attention on the behavior of interacting electrons. In Fig. 7 we present the variation of ground state energy and corresponding persistent current as a function of flux $\phi$ for an interacting 60-site Möbius strip in the half-filled band case $\left(N_{\uparrow}=N_{\downarrow}=30\right)$ for different values of $U$ considering $t=-1$ and $t_{\perp}=0$. It is found that with increasing the on-site Coulomb correlation strength $U$, ground state energy increases and its slope also gets changed though it 

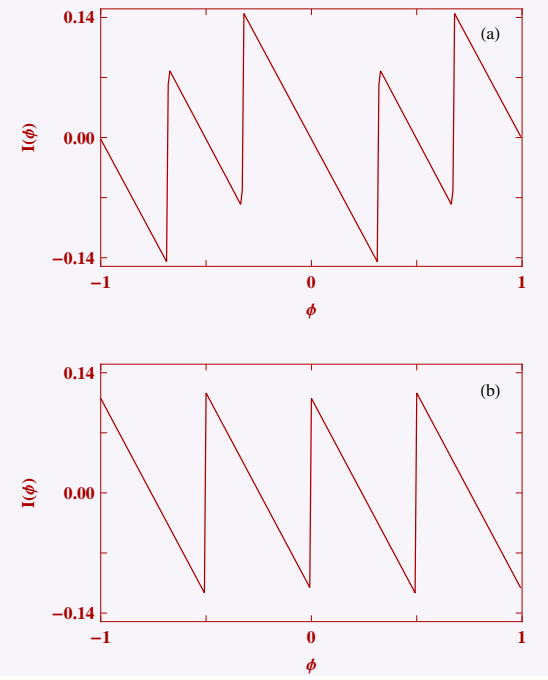

FIG. 5: (Color online). Current-flux characteristics of a 200site non-interacting $(U=0)$ Möbius strip considering $t=-1$ and $t_{\perp}=-0.8$, where (a) and (b) correspond to $N_{e}=95$ and 96 , respectively.

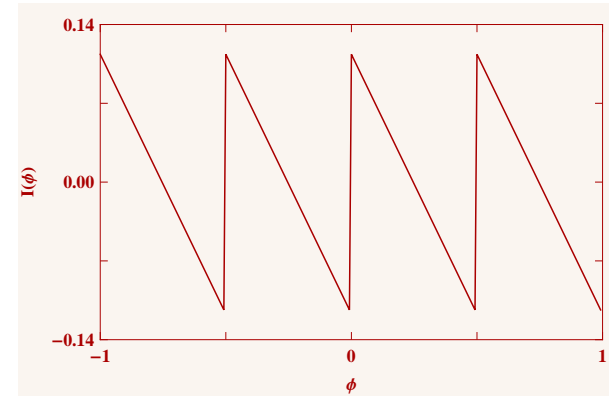

FIG. 6: (Color online). I- $\phi$ spectrum of a Möbius strip in the half-filled band case. The parameters are: $N=100, U=0$, $t=-1, t_{\perp}=-0.8$. Current exhibits $\phi_{0} / 2$ periodicity though $t_{\perp}$ is finite.

is not clear from the spectrum (Fig. [7(a)). This change in slope is nicely reflected in current-flux characteristics (Fig. [7(b)), where we see that current varies periodically with $\phi$ providing $\phi_{0} / 2$ periodicity and gets highly suppressed with $U$. At half-filling all atomic sites are occupied by single electrons having a particular spin (up or down) which do not allow opposite spin electrons in the same site due to repulsive Coulomb interaction. Thus, the electronic hopping or more precisely electronic mobility gets suppressed which yields reduced persistent current. In the large $U$ limit we practically get zero current i.e., the system becomes a Mott insulator.

Even in presence of $t_{\perp}$ current amplitude gets decreased with $U$, in the limit of half-filling, but the reduction of current is not as mush as we get in the absence of $t_{\perp}$. The additional hopping $\left(\mathrm{viz}, t_{\perp}\right.$ ) is responsible for it. This behavior is clearly noticed from the results given in Fig. 8 where we choose a 62 -site $(N=31)$ in-
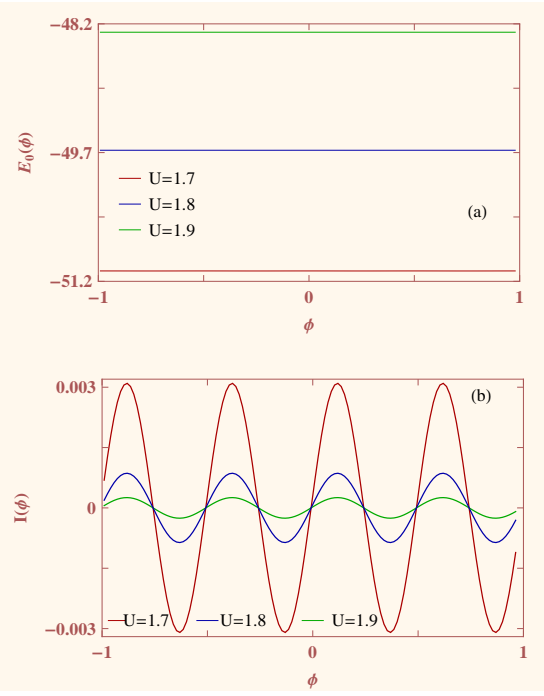

FIG. 7: (Color online). Dependence of ground state energy (upper panel) and corresponding current (lower panel) as a function of flux $\phi$ for a 60 -site interacting Möbius strip with $t=-1$ and $t_{\perp}=0$ for three different values of $U$. Here we choose $N_{\uparrow}=N_{\downarrow}=30$.
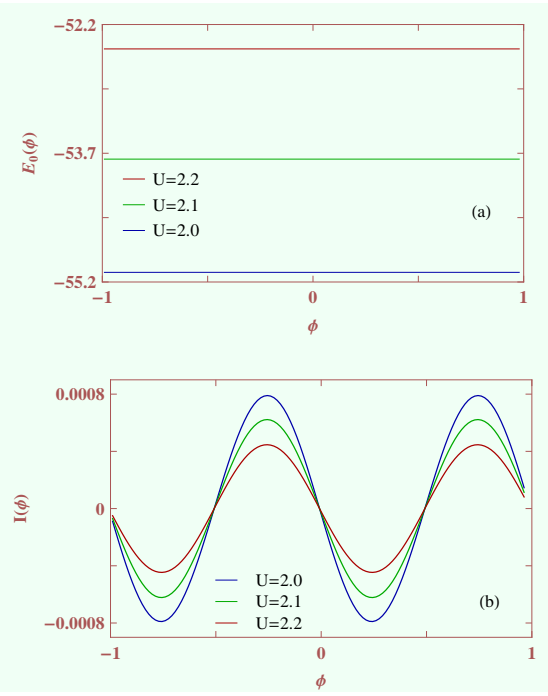

FIG. 8: (Color online). Ground state energy (upper panel) along with corresponding current (lower panel) as a function of $\phi$ for a 62 -site interacting Möbius strip with $t=-1$ and $t_{\perp}=-0.8$ for three different values of $U$. Here we choose $N_{\uparrow}=N_{\downarrow}=31$.

teracting Möbius strip with $N_{\uparrow}=N_{\downarrow}=31, t=-1$ and $t_{\perp}=-0.8$. For this geometry (with odd $N$ ) the current exhibits usual one-flux quantum $\left(\phi_{0}\right)$ periodicity as here we set a non-zero value of $t_{\perp}$. Whereas, an interacting half-filled Möbius strip with even $N$ exhibits unconventional half-flux quantum periodic current even though $t_{\perp}$ is finite (not shown here to save space), like what we get in the case of Möbius strip with non-interacting electrons (Fig. 6). 
The results presented in Fig. 7 and Fig. 8 are worked out for 60- and 62-site Möbius strips, respectively, which can be considered as ultra-small systems. To see the effect of interaction among electrons in realistic Möbius rings which are significantly larger in Fig. 9 we present the current-flux characteristics considering a 102-site system in the half-filled band case. Going through the spec-
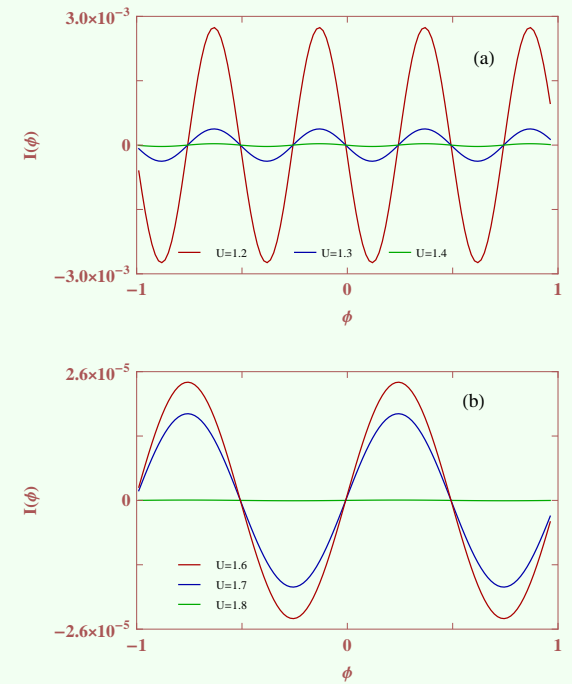

FIG. 9: (Color online). Current-flux characteristics for a 102site interacting Möbius strip in the half-filled band case at three different values of $U$ where (a) $t=-1, t_{\perp}=0$ and (b) $t=-1, t_{\perp}=-0.8$.

tra given in Figs. Z(b) and 8(b), it is clearly seen from Fig. 9 that the nature of periodicity and the suppression of current with $U$ remain exactly same for this $102-$ site Möbius strip, and the nature will be exactly identical even for much higher system sizes which we confirm through our detailed numerical calculation.

Now, in order to explain more clearly the interplay between on-site Hubbard interaction, hopping integrals, system size and filling factor we focus on the spectra given in Figs. 10 and 11, where the variation of typical current amplitude is shown. Taking the absolute value of maximum current within the range of one-flux quantum (viz, 0 to $\phi_{0}$ ) we determine this typical current $I_{\text {typ }}$. Figure 10 displays $I_{\mathrm{typ}}-U$ characteristics for different values of $t$ in the half-filled and less than half-filled band cases for a 60 -site Möbius strip. The other hopping integral i.e., $t_{\perp}$ is fixed at zero. At half-filling current starts decreasing when the e-e interaction is introduced, whereas for less than half-filled case it $\left(I_{\text {typ }}\right)$ remains almost constant for a specific $U$-window and then decreases with $U$. These features are essentially controlled by two competing parameters $t$ and $U$. In the limit of half-filling each site of the system is occupied by an electron and thus movement of electrons is not favorable due to repulsive interaction $U$ which results current reduction. While, the presence of empty sites in less than half-filled system allows electrons to hop from one site to other in the low $U$ limit where
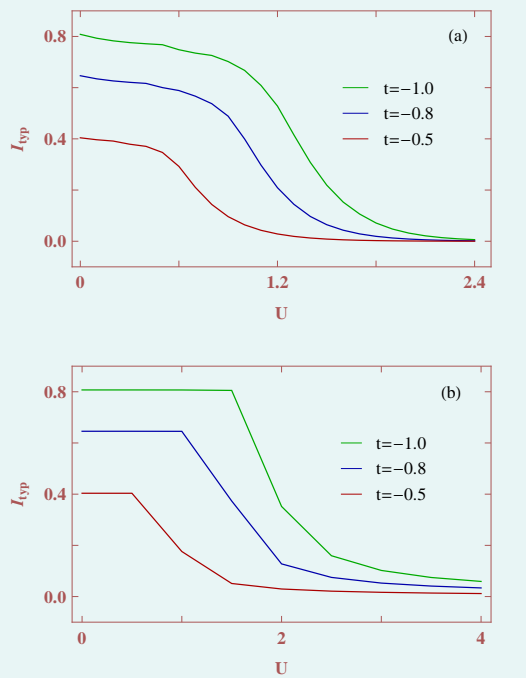

FIG. 10: (Color Online). $I_{\mathrm{typ}}-U$ characteristics for different values of $t$, setting $t_{\perp}=0$, for a 60-site Möbius strip, where (a) and (b) correspond to $N_{\uparrow}=N_{\downarrow}=30$ and $N_{\uparrow}=N_{\downarrow}=29$, respectively.
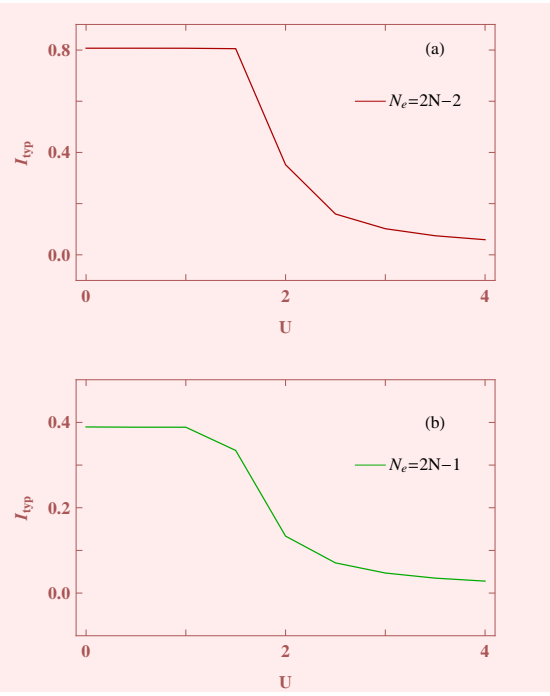

FIG. 11: (Color Online). Dependence of typical current amplitude $I_{\text {typ }}$ as a function of $U$ for four different band fillings considering a 60 -site $(N=30)$ Möbius strip with $t=-1$ and $t_{\perp}=0$, where (a) and (b) correspond to even and odd $N_{e}$, respectively. For even $N_{e}$ we set $N_{\uparrow}=N_{\downarrow}$, while for odd $N_{e}$ we choose $N_{\uparrow}=N_{\downarrow}+1$.

the hopping integral $t$ dominates over $U$ and makes the current almost $U$ independent. Beyond a critical $U$ repulsive interaction dominates and current starts decreasing. Eventually it reaches nearly to zero for large $U$. The rate of fall of current amplitude as well as the critical value of $U$ strongly depend on the filling factor, when the hopping integral remains constant, which is clearly seen from the spectra given Fig. 11] where (a) and (b) correspond to the even and odd $N_{e}$, respectively. These 
features can be well understood from the above analysis. Both for Figs. 10 and 11] we compute the results setting $t_{\perp}=0$. Exactly similar features are also obtained even when $t_{\perp} \neq 0$ and that is why we do not present those results to save space.

\section{B. Finite temperature limit}

This sub-section discusses the effect of temperature on current-flux characteristics for both non-interacting and interacting Möbius geometries. In Fig. 12 we present

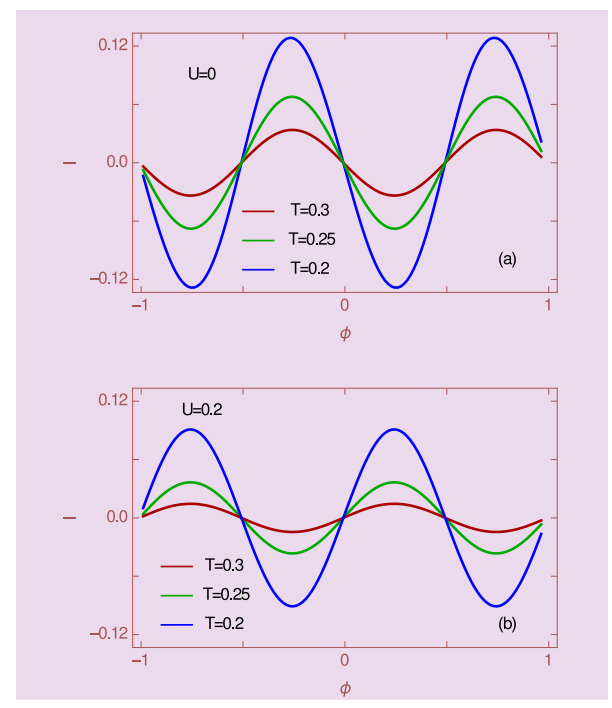

FIG. 12: (Color Online). Current-flux characteristics for a 60-site Möbius strip at three typical temperatures, where (a) and (b) correspond to $U=0$ and 0.2 , respectively. The other physical parameters are: $t=-1, t_{\perp}=-0.6$ and $\mu=0.25$.

the variation of persistent current as a function of flux $\phi$ for three distinct temperatures considering a 60-site Möbius strip, where (a) and (b) correspond to the noninteracting $(U=0)$ and interacting $(U=0.2)$ cases, respectively. From the spectra it is observed that the current decreases with system temperature. At finite temperatures, all energy levels contribute to current in certain percentage characterized by Fermi-Dirac distribution function. With increasing the temperature occupation probabilities of higher energy levels get increased and currents carried by successive energy levels in opposite directions are almost identical so that they mutually cancel each other which results a smaller net current. Certainly much lesser current is expected at higher temperatures. The reduction of current due to repulsive Coulomb interaction (shown from the spectra given in Fig. 12) remains same as discussed earlier.

Finally, to explore the asymptotic behavior of current with temperature we concentrate on the results presented in Fig. 13. The variation of typical current $I_{\text {typ }}$ as a function of temperature $T$ is given for a non-interacting spinless Möbius strip with 60 atomic sites considering

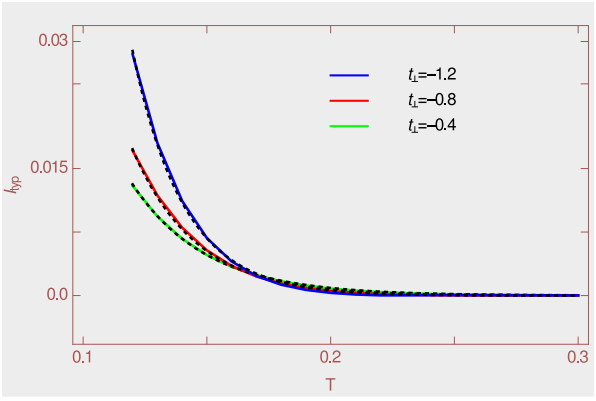

FIG. 13: (Color Online). Dependence of $I_{\text {typ }}$ with system temperature $T$ for a spinless Möbius strip $(N=30)$ at three different values of $t_{\perp}$, setting $t$ and $\mu$ at -1 and 0.25 , respectively.

$\mu=0.25$ and $t=-1$. Three different cases are analyzed depending on $t_{\perp}$, where the colored dotted points are computed from our theoretical prescription given in Sec. II. Using these dots we find a functional relation between $I_{\text {typ }}$ and temperature $T$ which looks like $I_{\text {typ }}=a \exp (-b N T)$, where the constants $a$ and $b$ depend on $t_{\perp}$. For $t_{\perp}=-0.4, a=0.74$ and $b=1.12$, and these values are 2 and 1.32 respectively for $t_{\perp}=-0.8$, and for $t_{\perp}=-1.2$, these constant factors are $a=9.5$ and $b=1.61$. Plotting this functional form we get the continuous curve, and we see that each curve, associated with $t_{\perp}$, matches extremely well with the dotted points. In this figure (Fig. 13) we present the results for a particular system size, but this exponential relation is absolutely general for any Möbius strip size which we confirm through our detailed numerical analysis. Only the factors $a$ and $b$, associated with $t_{\perp}$, get changed. In addition, it is important to note that even for interacting Möbius strip we find exactly identical functional relation of typical current with temperature $T$.

Accuracy of MF calculations: To make the present communication a self contained study, at the end, we would like to discuss about the accuracy of the meanfield calculations in our geometry. We make a comparative study by calculating persistent current in two different ways. In one way we use Hartree-Fock mean field technique, and in the other way current is calculated by determining energy eigenvalues through exact diagonalization of the full many-body Hamiltonian Eq. 1] As the dimension of the Hamiltonian matrix increases sharply with system size as well as up and down spin electrons, we restrict ourselves to a small system size due to our computational limitations in diagonalization. The results are presented in Fig. 14. The currents shown by black dotted lines are computed by exact numerical diagonalization method, while the MF results are shown by red curves. We see that MF results match very well with the exact diagonalization technique. Here the currents are compared setting the system temperature at absolute zero. Similar agreement is also obtained for finite temperature, and thus, one can safely use HF mean-field 

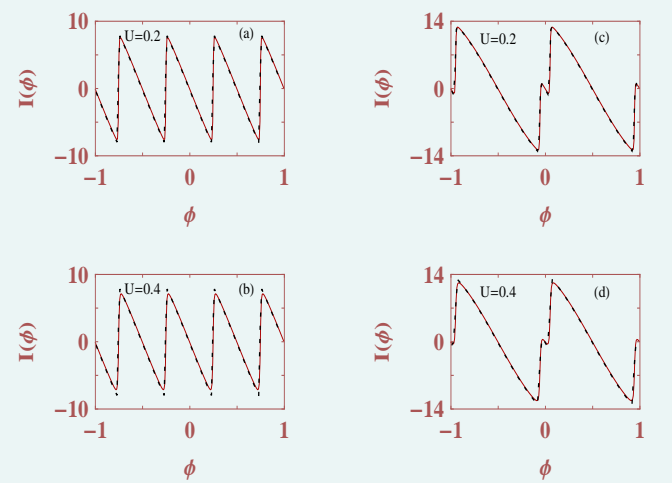

FIG. 14: (Color Online). Comparison between mean-field approach (black dotted line) and exact diagonalization method (red line) is performed. Current-flux characteristics are computed for a 6 -site Möbius strip for two distinct values of $U$ in the limit of zero temperature, where the 1st column corresponds to $t_{\perp}=0$ and it is -0.8 for the 2 nd column. Here we set $t=-1$.

approach to investigate magnetic response in our twisted ring geometry.

\section{CLOSING REMARKS}

In summary, we have investigated magnetic response of non-interacting and interacting electrons in a one-fold twisted Möbius strip subjected to an AB flux $\phi$. For the non-interacting system we have calculated energy eigenvalues and the corresponding current completely analytically, and under a typical case (viz, $t_{\perp}=0$ ) net current gets a closed form within a specific flux window. For all odd $N_{e}$ it exhibits one particular relation, and similarly, for all even $N_{e}$ it follows another relation. On the other hand, Hartree-Fock mean field theory has been utilized to study magnetic response of interacting electrons.

The essential findings are as follows. (i) Appearance of half-flux quantum $\left(\phi_{0} / 2\right)$ periodicity when the vertical hopping between two ring-channels is restricted i.e., $t_{\perp}=0$. (ii) Even for non-zero value of $t_{\perp}, \phi_{0} / 2$ periodic current can be observed if the system becomes halffilled and $N$ is even. (iii) Current is highly sensitive to the system temperature. It has been observed that, for a fixed system size, the typical current amplitude decreases sharply with increasing temperature $T$ following an exponential relation of the form $I_{\text {typ }}=a \exp (-b N T)$, irrespective of the e-e correlation strength.

In the present model we have ignored the effect of disorder. The interplay between Hubbard interaction and disorder on persistent current has already been discussed in several studies $11-13,41$, though mostly they are confined with simple loop geometries. Analogous behavior is also expected in Möbius geometry, but a deeper insight into this problem is very essential for further understanding. At the same time we have also ignored the effect of electron-phonon interaction since it does not provide any significant change in current in the said temperature regime.

Lastly we would like to state that all the features studied in this article can be utilized to explore magnetic response in other non-trivial topological systems.

\section{ACKNOWLEDGMENT}

MS is thankful to University Grants Commission, India for research fellowship.
* Electronic address: santanu.maiti@isical.ac.in

1 S. Tanda, T. Tsuneta, Y. Okajima, K. Inagaki, K. Yamaya, and N. Hatakenaka, Nature (London) 417, 397 (2002).

2 Z. L. Guo, Z. R. Gong, H. Dong, and C. P. Sun, Phys. Rev. B 80, 195310 (2009).

3 J. Gravesen and M. Willatzen, Phys. Rev. A 72, 032108 (2005).

4 E. H. M. Ferreira, M. C. Nemes, M. D. Sampaio, and H. A. Weidenmüller, Phys. Lett. A 333, 146 (2004).

5 M. Hayashi, H. Ebisawa, and K. Kuboki, Phys. Rev. B 72, 024505 (2005).

${ }^{6}$ E. L. Starostin and G. H. M. van der Heijden, Nat. Mater. 6, 563 (2007).

7 E. L. Starostin and G. H. M. van der Heijden, J. Elast. 119, 67 (2015).

8 M. Büttiker, Y. Imry, and R. Landauer, Phys. Lett. A 96, 365 (1983).

9 H. F. Cheung, Y. Gefen, E. K. Reidel, and W. H. Shih, Phys. Rev. B 37, 6050 (1988).

${ }^{10}$ H. F. Cheung, E. K. Riedel, and Y. Gefen, Phys. Rev. Lett.
62, 587 (1989).

11 G. Montambaux, H. Bouchiat, D. Sigeti, and R. Friesner, Phys. Rev. B 42, 7647 (1990).

12 G. Bouzerar, D. Poilblanc, and G. Montambaux, Phys. Rev. B 49, 8258 (1994).

13 T. Giamarchi and B. S. Shastry, Phys. Rev. B 51, 10915 (1995).

14 S. K. Maiti, J. Chowdhury and S. N. Karmakar, Phys. Lett. A 332, 497 (2004).

15 S. K. Maiti, Solid State Phenomena 155, 87 (2009).

16 M. Saha and S. K. Maiti, Phys. Lett. A 380, 1450 (2016).

17 V. Ambegaokar and U. Eckern, Phys. Rev. Lett. 65, 381 (1990).

18 A. Schmid, Phys. Rev. Lett. 66, 80 (1991).

19 I. V. Sankar, P. J. Monisha, S. Sil, and A. Chatterjee, Physica E 73, 175 (2015).

20 H. Bary-Soroker, O. Entin-Wohlman, and Y. Imry, Phys. Rev. B 82, 144202 (2010).

21 L. P. Lévy, G. Dolan, J. Dunsmuir, and H. Bouchiat, Phys. Rev. Lett. 64, 2074 (1990). 
22 E. M. Q. Jariwala, P. Mohanty, M. B. Ketchen, and R. A. Webb, Phys. Rev. Lett. 86, 1594 (2001).

23 N. O. Birge, Science 326, 244 (2009).

24 V. Chandrasekhar, R. A. Webb, M. J. Brady, M. B. Ketchen, W. J. Gallagher, and A. Kleinsasser, Phys. Rev. Lett. 67, 3578 (1991).

25 W. Rabaud, L. Saminadayar, D. Mailly, K. Hasselbach, A. Benoit, and B. Etienne, Phys. Rev. Lett. 86, 3124 (2001).

26 D. Mailly, C. Chapelier, and A. Benoit, Phys. Rev. Lett. 70, 2020 (1993).

27 H. Bluhm, N. C. Koshnick, J. A. Bert, M. E. Huber, and K. A. Moler, Phys. Rev. Lett. 102, 136802 (2009).

${ }^{28}$ V. M. Fomin, Physics of Quantum Rings, Springer-Verlag Berlin Heidelberg (2014).

29 D. Rai, O. Hod, and A. Nitzan, J. Phys. Chem. C 114, 20583 (2010).

30 S. K. Maiti, Eur. Phys. J. B 86, 296 (2013).

31 D. Rai, O. Hod, and A. Nitzan, Phys. Rev. B 85, 155440 (2012).

32 S. K. Maiti, J. Appl. Phys. 117, 024306 (2015).
${ }^{33}$ K. Yakubo, Y. Avishai and D. Cohen, Phys. Rev. B 67, 125319 (2003).

34 H. Mori and R. Ota, J. Phys.: Conf. Ser. 150, 022058 (2009).

35 B. Lassen, M. Willatzen, and J. Gravesen, J. Nanoelectron. Optoelectron. 6, 68 (2011).

36 Z. Li and L. R. Ram-Mohan, Phys. Rev. B 85, 195438 (2012).

37 V. M. Fomin, S. Kiravittaya, and O. G. Schmidt, Phys. Rev. B 86, 195421 (2012).

38 H. Kato and D. Yoshioka, Phys. Rev. B 50, 4943 (1994).

39 A. Kambili, C. J. Lambert, and J. H. Jefferson, Phys. Rev. B 60, 7684 (1999).

40 S. K. Maiti and A. Chakrabarti, Phys. Rev. B 82, 184201 (2010).

41 M. Saha and S. K. Maiti, Physica E 84, 118 (2016).

42 S. Gupta, S. Sil, and B. Bhattacharyya, Physica B 355 , 299 (2005). 